\title{
A fermented milk concentrate and a combination of short-chain galacto-oligosaccharides/long-chain fructo-oligosaccharides/pectin-derived acidic oligosaccharides protect suckling rats from rotavirus gastroenteritis
}

\author{
Mar Rigo-Adrover ${ }^{1,2}$, Teresa Pérez-Berezo ${ }^{1,2}$, Sara Ramos-Romero ${ }^{1,2}$, Kees van Limpt ${ }^{3}$, \\ Karen Knipping ${ }^{3,4}$, Johan Garssen ${ }^{3,4}$, Jan $\mathrm{Knol}^{3}$, Àngels Franch ${ }^{1,2}$, Margarida Castell ${ }^{1,2}$ and \\ Francisco J. Pérez-Cano ${ }^{1,2 *}$ \\ ${ }^{1}$ Departament de Bioquímica i Fisiologia, Facultat de Farmàcia i Ciències de l'Alimentació, University of Barcelona (UB), \\ o8028 Barcelona, Spain \\ ${ }^{2}$ Institut de Recerca en Nutrició i Seguretat Alimentària (INSA), 08921 Santa Coloma de Gramenet, Spain \\ ${ }^{3}$ Nutricia Research, 3584 CT Utrecht, The Netherlands \\ ${ }^{4}$ Division of Pharmacology, Utrecht Institute for Pharmaceutical Sciences, Faculty of Science, Utrecht University, 3584 CG \\ Utrecht, The Netherlands
}

(Submitted 27 July 2016 - Final revision received 28 October 2016 - Accepted 9 December 2016 - First published online 7 Febraury 2017)

\section{Abstract}

Human milk contains bioactive compounds that confer a protective role against gastrointestinal infections. In order to find supplements for an infant formula able to mimic these benefits of breast-feeding, two different concepts were tested. The products consisted of the following: (1) a Bifidobacterium breve- and Streptococcus thermophilus-fermented formula and (2) a combination of short-chain galacto-oligosaccharides/ long-chain fructo-oligosaccharides with pectin-derived acidic oligosaccharides. A rotavirus infection suckling rat model was used to evaluate improvements in the infectious process and in the immune response of supplemented animals. Both nutritional concepts caused amelioration of the clinical symptoms, even though this was sometimes hidden by softer stool consistency in the supplemented groups. Both products also showed certain modulation of immune response, which seemed to be enhanced earlier and was accompanied by a faster resolution of the process. The viral shedding and the in vitro blocking assay suggest that these products are able to bind the viral particles, which can result in a milder infection. In conclusion, both concepts evaluated in this study showed interesting protective properties against rotavirus infection, which deserve to be investigated further.

Key words: Prebiotics: Fructo-oligosaccharides: Galacto-oligosaccharides: Pectin-derived acidic oligosaccharides: Fermented formula: Milk: Rotavirus

The WHO refers to breast-feeding as the best way of providing food and promoting the healthy development of infants ${ }^{(1)}$. In fact, human milk is not only a source of energy and nutrients but also contains bioactive compounds. These compounds such as oligosaccharides, nucleotides, growth factors, hormones, Ig, cytokines, lysozyme and lactoferrin have shown immunomodulatory and bifidogenic effects ${ }^{(2-4)}$. One of the main benefits of breast-feeding is the protection that it confers against gastrointestinal infections in both developed and developing countries $^{(2)}$. Despite this, breast-feeding is not always possible; thus, the formulation of new infant formulae, which better mimic the composition of human milk and provide its antiinfectious components, is of great importance. In this sense, dietary oligosaccharide structures, which have a prebiotic effect similar to that of human milk oligosaccharides, are usually added to infant formulae. Interventional studies with a specific mixture of short-chain galacto-oligosaccharides (scGOS) and long-chain fructo-oligosaccharides (lcFOS; 9:1 ratio) have been shown to induce a microbiota composition and a stool consistency closer to the breast-fed infant pattern ${ }^{(5,6)}$. In addition, scGOS and lcFOS supplements produce an increase in SCFA in caecum samples ${ }^{(7,8)}$, an increase in intestinal IgA secretion $^{(9,10)}$, an improvement in Ca absorption ${ }^{(11,12)}$, a lower incidence of infections during the first few years of life ${ }^{(5,13,14)}$ and also a decreased incidence of food allergy, asthma and eczema $^{(15)}$ in both clinical and preclinical studies. All these effects suggest the importance of scGOS and lcFOS in early life. Recently, prebiotics other than FOS and GOS have been tested

Abbreviations: FM, fermented milk; GFA, scGOS/lcFOS/pAOS mixture; lcFOS, long-chain fructo-oligosaccharides; pAOS, pectin-derived acidic oligosaccharides; REF, reference; RV, rotavirus; scGOS, short-chain galacto-oligosaccharides.

* Corresponding author: F. J. Pérez-Cano, fax +34 9340359 01, email franciscoperez@ub.edu 
in animals and in humans, such as pectin-derived acidic oligosaccharides (pAOS). The pAOS, whose safety has already been assessed ${ }^{(16)}$, are tested in combination with scGOS/lcFOS in order to mimic the presence of neutral and acidic oligosaccharides in human milk. This type of mixture has been shown to have a bifidogenic effect ${ }^{(17)}$. Moreover, it has been demonstrated to have an influence on the immune system by enhancing Th1 response, which is weak in early life, in an infection model and in a vaccination mouse model ${ }^{(18,19)}$, as well as in a food allergy model $^{(20)}$. In infants, this mixture also reduces the occurrence of atopic dermatitis ${ }^{(21)}$. Some studies point out that this combined formula shows more pronounced immunomodulatory effects than scGOS/lcFOS alone ${ }^{(19)}$. Therefore, differential mechanisms of action have been suggested ${ }^{(20)}$.

Besides prebiotic- or probiotic-containing formulae, fermented milk (FM)-based infant formulae (FMBIF) offer an additional means for modulating gut immunity and/or gut microbiota. These formulae are produced by the fermentation of cows' milk with specific lactic acid bacteria strains, followed by heat treatment. These formulae contain specific products resulting from the fermentation process. In addition to the possible modulation of gut microbiota composition, in vitro and in vivo studies suggest that specific fermentation products can actively participate in the establishment of immune balance and oral tolerance ${ }^{(22)}$. FMBIF have also been shown to have a bifidogenic effect associated with an increase in intestinal IgA secretion and a decrease in faecal $\mathrm{pH}^{(23,24)}$. FMBIF have also demonstrated their ability to reduce the severity of rotavirus (RV) gastrointestinal infection ${ }^{(25,26)}$

The present study aimed to elucidate the immunomodulatory and the protective effects of a mixture of scGOS/lcFOS/pAOS and heat-treated (probiotic-) FM components against RV infection in early life. A neonatal rat model of RV gastroenteritis was applied $^{(4,27,28)}$ because RV is the most common pathogen causing diarrhoea among infants and young children ${ }^{(29)}$.

\section{Methods}

\section{Animals}

A total of twelve G14 pregnant Lewis rats were obtained from Janvier (Le-Genest-Saint-Isle, France) and allowed to deliver at term. The day of birth was registered as day 1 of life. Litters were unified to $7 \mathrm{pups} / \mathrm{dam}$ and had free access to nipples. Dams had access to water and the American Institute of Nutrition (AIN)-93G diet ${ }^{(30)}$ ad libitum. Each dam and its litter were housed individually under controlled temperature and humidity conditions in a $12 \mathrm{~h}$ light $-12 \mathrm{~h}$ dark cycle. Furthermore, they were maintained in a safe, isolated room, specially designed and authorised for working under biosecurity level 2 conditions at the Animal Service of the Faculty of Pharmacy, University of Barcelona. Pups were individually identified by labelling with a permanent marker after $2 \mathrm{~d}$ of environmental adaptation. The animals were weighed and monitored daily in order to obtain data regarding the influence of virus inoculation, clinical development and nutritional intervention on body weight and growth.

This study was conducted in accordance with institutional guidelines for the care and use of laboratory animals established by the Ethics Committee for Animal Experimentation of the University of Barcelona and the Catalonian Government (CEEA-UB ref. 165/11, DAAM: 5871).

\section{Experimental design}

Suckling rats were distributed into four different experimental groups: reference (REF group), the group receiving RV inoculation (RV group), the group receiving heat-treated (probiotic-) FM components (FM group) and the one receiving scGOS/ lcFOS/pAOS mixture (GFA group). Each group included three litters with seven pups each ( $n$ 21/group). Diets or vehicle were administered from day 3 of life until the last day of the experiment. The RV inoculation was carried out at day 7 of age in all the experimental groups with the exception of the REF group. Clinical evaluation was performed daily from the day before inoculation until the end of the study. Faecal samples were collected daily throughout the study, and blood and intestinal wash samples were collected on the day animals were killed. Animals were euthanised at two different time points: a group of the animals were euthanised on day 14 ( $n$ 9), whereas the others continued receiving the diet until day 21 ( $n$ 12). A parallel cohort with non-infected animals receiving the products was also included (non-infection study (NIS)).

\section{Dietary supplementation}

Animals were administered daily by oral gavage from day 3 to day 21 of age with the different supplements (two groups: FM and GFA) or vehicle (two groups: RV and REF), as previously described $^{(31)}$, using low-capacity syringes (Hamilton Bonaduz) adapted to forced alimentation tubes of 25 or 23 calibre and $27 \mathrm{~mm}$ in length (ASICO).

The FM diet was obtained by fermentation with Bifidobacterium breve $\mathrm{C} 50\left(4.2 \times 10^{9}\right.$ bacteria/100 g of powder formula) and Streptococcus thermophilus $065\left(3.84 \times 10^{7}\right.$ bacteria/100 g of powder formula) during the manufacturing process and then heated to kill ferments (patented technology; Blédina). It was administered at a dose of $3 \mathrm{~g} / 100 \mathrm{~g}$ of body weight per $d$. The GFA group received a combination of scGOS, lcFOS and pAOS $(76 \cdot 5: 8 \cdot 5: 15)$ at a dose of $0 \cdot 8 \mathrm{~g} / 100 \mathrm{~g}$ of body weight per $\mathrm{d}$.

\section{Virus inoculation}

The RV strain used (simian SA-11) was obtained from the 'Enteric Virus Group' of the University of Barcelona. Viruses were propagated in fetal African green monkey kidney cells (MA-104) and titred as TCID $_{50} / \mathrm{ml}$ (TCID, tissue culture infectious dose $)^{(4,27)}$. Production was carried out in compliance with the current principles of Good Laboratory Practices (GLP) (Royal Decree 1369/2000, of 19 July). SA-11 was inoculated $\left(2 \times 10^{8} \mathrm{TCID}_{50} /\right.$ rat in $100 \mu \mathrm{l}$ of PBS) at day 7 of life, as previously described $^{(27)}$, to suckling rats from the RV, FM and GFA groups. The RV was inoculated after $1 \mathrm{~h}$ of separation from their dams to avoid interference between RV and milk components. The REF group received the same volume of PBS under the same conditions. 


\section{Clinical indices and faecal specimen collection}

SA-11 infection was evaluated on days $8-21$ by the growth rate and clinical indices derived from faecal samples as previously described $^{(27)}$. Faecal sampling was performed once per $\mathrm{d}$ by gently pressing and massaging the abdomen. Specimens were immediately scored, in a blinded manner, weighed and frozen at $-20^{\circ} \mathrm{C}$ for further analysis.

Severity of diarrhoea was expressed by faecal weight and by scoring stools from 1 to 4 (diarrhoea index) on the basis of colour, texture and amount as follows: normal (diarrhoea index $=1$ ), loose yellow-green (diarrhoea index $=2$ ), totally loose yellowgreen (diarrhoea index $=3$ ) and high amount of watery (diarrhoea index $=4$ ) faeces. Diarrhoea scores $\geq 2$ indicate diarrhoeic faeces, whereas scores $<2$ indicate absence of diarrhoea.

The AUC of severity during days $7-13$ was calculated as a global value of severity. The maximum diarrhoea index was defined as the highest score during the diarrhoea period. Incidence of diarrhoea was expressed by the percentage of diarrhoeic animals, taking into account the number of animals in each group, and by the percentage of diarrhoeic faeces, taking into consideration the number of total samples collected every day in each group. The AUC of the percentage of diarrhoeic animals and the percentage of diarrhoeic faeces curves during days 7-13 were calculated as a global value of incidence.

The AUC for severity, percentage of diarrhoeic animals and percentage of diarrhoeic faeces were also calculated taking into account the basal values because of intrinsic aspects of each treatment (normalised AUC). The maximum percentage of diarrhoeic animals and diarrhoeic faeces were defined as the highest values during the diarrhoea period. The diarrhoea period was calculated for each animal as the interval between the first (beginning day of diarrhoea) and the last day (ending day of diarrhoea) of diarrhoea. The actual number of days with diarrhoea within the diarrhoea period was also counted.

\section{Faecal pH determination}

Frozen faecal samples $(5-60 \mathrm{mg}$ ) from days 8-12 were diluted in distilled water (up to $200 \mathrm{mg} / \mathrm{ml}$ ) and softly agitated. Their $\mathrm{pH}$ values were measured by a $\mathrm{pH}$ electrode for surfaces 5207 (Crison Instruments) and pH-Meter micropH 2001 (Crison Instruments), which were previously calibrated.

\section{Intestinal and blood sample collection}

After previous anaesthesia with intramuscular ketamine/xylazine injection, rats from each group were euthanised at days 14 or 21 . Blood samples were collected by cardiac puncture, and serum samples were stored at $-20^{\circ} \mathrm{C}$ until analysis. The small intestine was weighed, cut into 5-mm pieces and incubated in PBS for $10 \mathrm{~min}$ at $37^{\circ} \mathrm{C}$ in a shaker to obtain the gut wash. After centrifugation, supernatants were stored at $-20^{\circ} \mathrm{C}$ until analysis.

\section{ELISA for specific total, IgG, IgA and IgM anti-rotavirus antibody quantification in sera and intestinal washes}

Ninety-six-well plates (Nunc MaxiSorp) were coated with UV-inactivated SA-11 particles at $10^{5} / \mathrm{ml}$. After blocking with
PBS-1\%, bovine serum albumin (BSA, $1 \mathrm{~h}$, room temperature (RT)), appropriately diluted sera or intestinal wash samples were added (3h, RT). After washing, rabbit anti-rat Ig conjugated to peroxidase from Dako or mouse biotinylated anti-rat IgA (A93-2), IgG1/2a (R19-15) or IgM (G53-238) monoclonal antibodies from BD Biosciences, followed by peroxidase-conjugated extravidin (Sigma-Aldrich), were added. Subsequently, the substrate was added, as previously described $^{(27)}$. Pooled sera from dams of inoculated litters were used as standard in each plate. Quadratic polynomial adjustment was used. Dam sera received the value of 5000 arbitrary units (AU)/ml for total anti-RV antibodies and $1000 \mathrm{AU} / \mathrm{ml}$ for anti-RV IgG, IgA and IgM antibodies.

\section{ELISA for determining SA-11 viral load in faeces}

Faecal samples from days 7 to 16 were diluted in PBS $(20 \mathrm{mg} / \mathrm{ml})$ and homogenised using a Polytron (Kinematica). Homogenates were centrifuged $\left(200 \mathrm{~g}, 5 \mathrm{~min}, 4^{\circ} \mathrm{C}\right)$, and supernatants were frozen at $-20^{\circ} \mathrm{C}$ until use. SA-11 particles in faecal samples were quantified by ELISA, as previously described $^{(27)}$. Titrated dilutions of inactivated SA-11 virus particles, ranging from $10^{5}$ to $10^{3} / \mathrm{ml}$, were used as standard in each plate.

\section{In vitro blocking assay}

A concentration of $5 \times 10^{4}$ particles/ml of SA-11 in PBS-Tween $1 \%$ was prepared. Different dilutions of FM or GFA products of the in vivo-administered concentration were added to the virus (from $1 / 2$ to $1 / 60$ dilutions). The combinations were incubated for $30 \mathrm{~min}$. Free, non-coated viral particles were quantified by ELISA, as described above (SA-11 viral load).

\section{Statistical analysis}

PASW Statistics 22 software package (SPSS Inc.) was used for the statistical analysis. The Komolgorov-Smirnov test was applied to assess normal distribution, followed by Levene's test in order to determine variance equality. Conventional one-way ANOVA was performed considering the experimental group as the independent variable. When virus inoculation/treatment had a significant effect on the dependent variable (body weight, body weight increase, etc.), Scheffé's post hoc test was applied. The Kruskal-Wallis and Mann-Whitney $U$ tests were used when non-normal distribution or different variance was found (maximum diarrhoea score, severity AUC, etc.). Finally, the $\chi^{2}$ test was used to compare frequencies (diarrhoea incidence). Differences were considered significant at $P$ values $<0.05$. Results are expressed as means values with their standard errors or as medians and interquartile range of number of animals.

\section{Results}

Effect of the dietary supplementation and virus inoculation on body weight

Body weight was recorded between days 2 and 14 of life. The values ranged from about $6-7$ to $22-24 \mathrm{~g}$, with no significant 


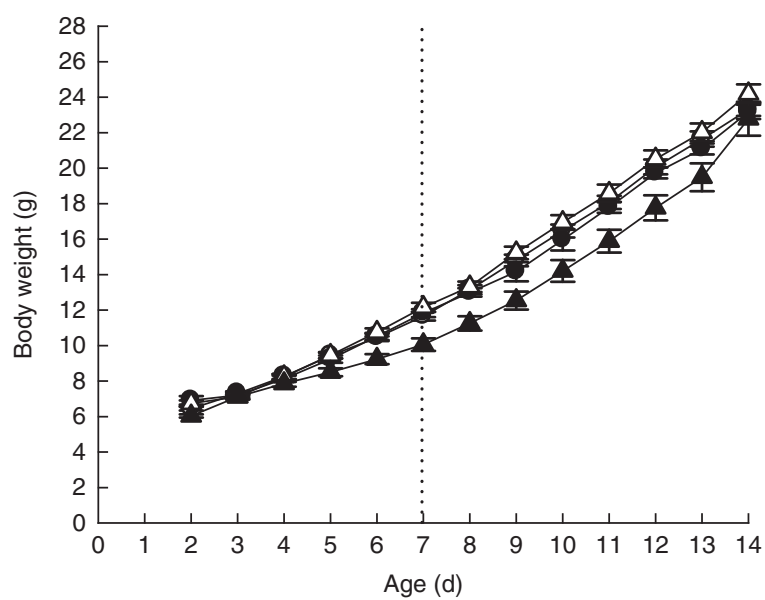

Fig. 1. Body weight (g) during the study, before and after virus inoculation on day 7. Values are means ( $n 21$ animals per group), with their standard errors. Statistical significance is explained in the text. -0 , Reference (REF group); $\longrightarrow$ - rotavirus (RV group); $\_$, fermented milk (FM group); $\_$, shortchain galacto-oligosaccharides/long-chain fructo-oligosaccharides/pectinderived acidic oligosaccharides mixture (GFA group).

differences among groups. When weight increase (from day 2 to day 14) was calculated, all animals had an increase of about 195-255\% without statistical differences. The diet had no notable influence on growth of animals, and no weight loss was associated with the infection process (Fig. 1). Only the GFA group had a lower body weight slope than the other groups from day 6 to day $13(P<0.05)$, although at the end of the study no differences were found either in body weight or in weight increase.

\section{Effect of the dietary supplementation on stool consistency}

As can be seen in Fig. 2, before the inoculation day (day 7) and when infection was resolved (day 14), the diets induced changes in faecal consistency (mainly the GFA diet), increasing the number of faeces considered as diarrhoeic (diarrhoea index $\geq 2$ ). To better observe these effects, already described for certain prebiotics, a NIS including suckling rats that received FM and GFA diets was carried out. According to our diarrhoea scores, most of the animals in the NIS had scores higher than 1 during the study and in some cases had a diarrhoea index $=2$ score during the days of supplementation (14d) (data not shown). Moreover, some animals of the NIS, mainly those from the GFA diet, still had diarrhoea 1 week later (day 21). Therefore, the direct effect of the diets on stool consistency has to be taken into account.

\section{Incidence of diarrhoea}

The incidence of diarrhoea was evaluated by two approaches: percentage of diarrhoeic animals and percentage of diarrhoeic faeces. Considering the percentage of diarrhoeic animals in the entire experimental period (Fig. 2(a)), 95-100\% of animals of the inoculated groups developed diarrhoea at some time point, whereas $<5 \%$ did so in the REF group (with no RV inoculation). In the RV group, percentage of diarrhoeic animals was almost $70 \%$ on day 8 ; it increased up to $85 \%$ on day 10 , decreased later, and after day 12 none of the animals in this group had diarrhoea (Fig. 2(a)). The animals from the FM group showed certain improvement. In this sense, this group had lower percentage of diarrhoeic animals than the RV group over the days 9-11 period, but it was only significant on day $11(P<0.05$ $v$. RV) (Fig. 2(a)). The percentage of diarrhoeic animals in the GFA group was higher than that of the RV group in the last few days studied (from day 12), as well as before virus inoculation. When the percentage of diarrhoea was normalised (subtracting the basal values due to intrinsic aspects of each treatment), a clearer improvement by both supplements was observed (Fig. 2(b)).

All groups had the maximum percentage of diarrhoeic animals at 1-3 d after the RV induction (Table 1). When the diarrhoeic animals' AUC was calculated (Table 1), we observed that the FM group presented a lower value than the RV group. On the contrary, the diarrhoeic animals' AUC in the GFA group was higher than that in the RV group. However, when the diarrhoeic animals' AUC was normalised by calculating the AUC of the increment of incidence during RV infection from the baseline of each group, it was lower for both supplemented groups compared with that of the RV group.

The results corresponding to the incidence of diarrhoeic faeces (percentage of diarrhoeic faeces) (Table 1), that is, maximum percentage of diarrhoeic faeces, diarrhoeic faeces AUC and normalised diarrhoeic faeces AUC, followed the same pattern as that of percentage of diarrhoeic animals.

\section{Duration of diarrhoea}

Focusing on the duration of the diarrhoea process, in the RV group, diarrhoea started 1-2d after inoculation and ended around the 11th day of life. Both, the diarrhoea period and the days with diarrhoea were between 3 and $4 \mathrm{~d}$ (Table 1). In the FM group, the ending day of diarrhoea was earlier compared with the other groups, and also the diarrhoea period and days with diarrhoea were reduced by up to $1 \mathrm{~d}$ (Table 1 ). In contrast, the GFA group had significantly increased the length of the diarrhoea period when no normalisation was applied (Table 1). It should be noted that the GFA group still had scores $>1$ up to the end of the study.

\section{Severity of diarrhoea}

In Fig. 2(c), the severity curves can be observed. In the RV group, the diarrhoea index was about 2.5 on day 8 and maintained similar values until day 11 , but later the mean score was below 2, which means that most animals had no diarrhoea. The FM group showed a lower severity score than the RV group as early as day 8 , and this was maintained until day 12 , although these differences were only significant at day 10 and $11(P<0.05)$. As in the previous indicators of the pathology (percentage of diarrhoeic animals, percentage of diarrhoeic faeces or the diarrhoea process variables), the effectiveness of the GFA diet in controlling the RV infection was hidden because the products induced features that concealed their putative action, but when normalised a remarkable reduction in diarrhoea severity was observed (Fig. 2(d)).

The mean maximum diarrhoea index for all infected groups was approximately 3 and was obtained at day 9 in all 
(a)
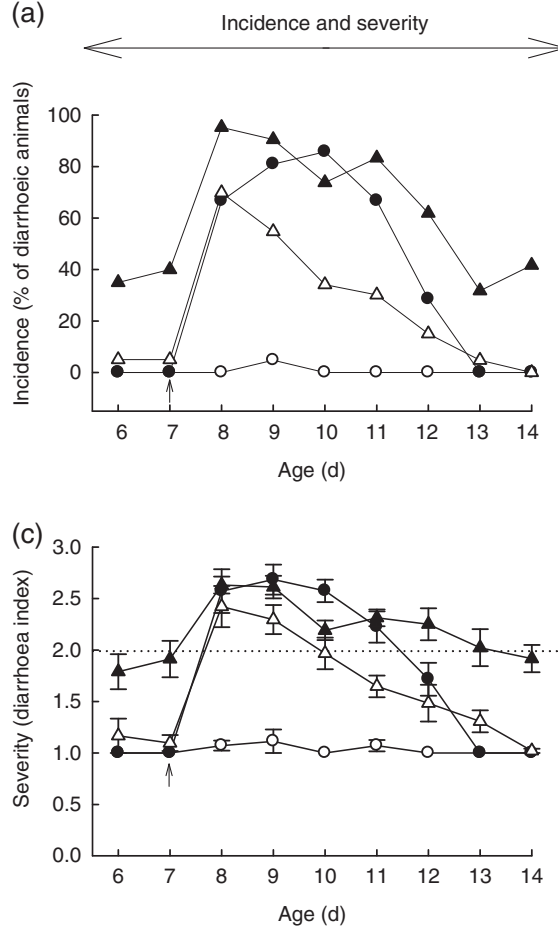

(b)
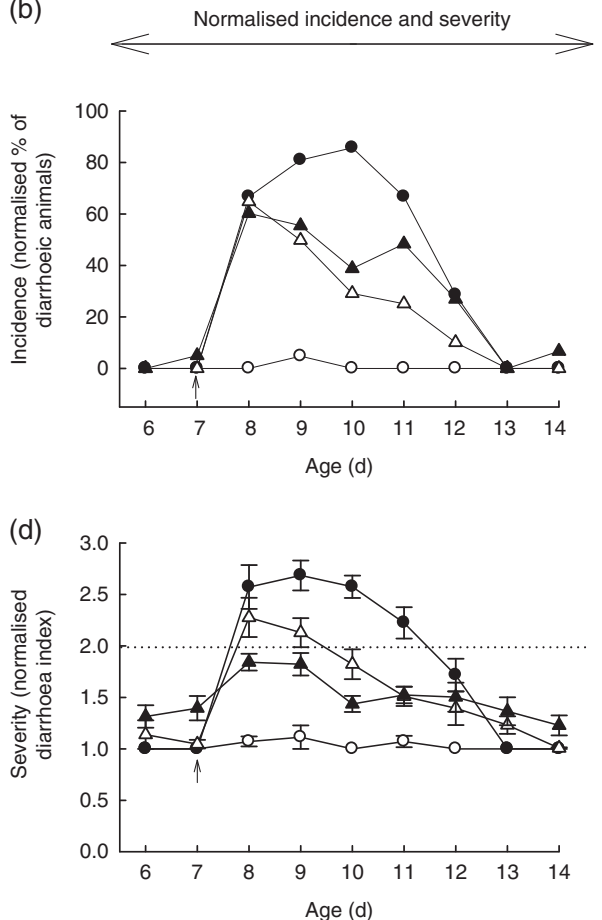

Fig. 2. Diarrhoea incidence. (a) Percentage of diarrhoeic animals. (b) Normalised percentage of diarrhoeic animals (subtracting the basal values due to intrinsic aspects of each treatment). (c) Severity of diarrhoea in a scale from 0 to 4 . Scores of diarrhoea index $\geq 2$ indicate diarrhoeic faeces. (d) Normalised severity of diarrhoea (subtracting the basal values due to intrinsic aspects of each treatment). Values are means ( $n 21$ animals per group), with their standard errors. Statistical significance is explained in the text. $\multimap-$, Reference (REF group); $\multimap-$, rotavirus (RV group); $\neg-$, fermented milk (FM group); $\longrightarrow$, short-chain galacto-oligosaccharides/ long-chain fructo-oligosaccharides/pectin-derived acidic oligosaccharides mixture (GFA group); $\uparrow, \mathrm{RV}$ inoculation day (day 7 of life).

Table 1. Clinical variables determining diarrhoea process from day 7 to 13 of life (Medians and interquartile ranges (IQR); $n 21$ animals per group)

\begin{tabular}{|c|c|c|c|c|c|c|}
\hline & \multicolumn{2}{|c|}{ RV } & \multicolumn{2}{|c|}{ FM } & \multicolumn{2}{|c|}{ GFA } \\
\hline \multicolumn{7}{|l|}{ Incidence } \\
\hline Maximum percentage of diarrhoeic animals & \multicolumn{2}{|c|}{$85 \cdot 71$} & \multicolumn{2}{|c|}{$70 \cdot 00$} & \multicolumn{2}{|c|}{$95 \cdot 00$} \\
\hline Diarrhoeic animals AUC & \multicolumn{2}{|c|}{328.57} & \multicolumn{2}{|c|}{$208 \cdot 85$} & \multicolumn{2}{|c|}{$440 \cdot 63$} \\
\hline Normalised diarrhoeic animals AUC & \multirow{2}{*}{\multicolumn{2}{|c|}{$\begin{array}{r}328.57 \\
90.00\end{array}$}} & \multirow{2}{*}{\multicolumn{2}{|c|}{$\begin{array}{r}178.97 \\
87.50\end{array}$}} & \multirow{2}{*}{\multicolumn{2}{|c|}{$232 \cdot 26$}} \\
\hline Maximum percentage of diarrhoeic faeces & & & & & \multirow{2}{*}{\multicolumn{2}{|c|}{511.67}} \\
\hline Diarrhoeic faeces AUC & \multicolumn{2}{|c|}{362.06} & \multicolumn{2}{|c|}{274.68} & & \\
\hline \multirow[t]{2}{*}{ Normalised diarrhoeic faeces AUC } & \multicolumn{2}{|c|}{$362 \cdot 06$} & \multicolumn{2}{|c|}{$183 \cdot 33$} & \multicolumn{2}{|c|}{$290 \cdot 61$} \\
\hline & Median & IQR & Median & IQR & Median & IQR \\
\hline \multicolumn{7}{|l|}{ Duration } \\
\hline Beginning day & $8 \cdot 0$ & 1.0 & $8 \cdot 0^{*}$ & $0 \cdot 0$ & $8 \cdot 0^{*}$ & 0.0 \\
\hline Ending day & $11 \cdot 0$ & $2 \cdot 0$ & 10.5 & $2 \cdot 0$ & $12 \cdot 0^{*, \dagger}$ & 1.0 \\
\hline Diarrhoea period & $4 \cdot 0$ & 1.0 & $2 \cdot 0^{*}$ & $2 \cdot 0$ & $5 \cdot 0^{*, \dagger}$ & 1.0 \\
\hline Days with diarrhoea & $4 \cdot 0$ & $2 \cdot 0$ & $2 \cdot 0^{*}$ & 1.0 & $5 \cdot 0^{*}, \dagger$ & 1.0 \\
\hline \multicolumn{7}{|l|}{ Severity } \\
\hline Maximum diarrhoea index & 3.00 & 0.25 & $2 \cdot 74^{\star}$ & 0.56 & 3.00 & 0.50 \\
\hline Severity AUC & 5.75 & 3.50 & $4 \cdot 31^{*}$ & 1.75 & $6 \cdot 88^{\dagger}$ & 2.69 \\
\hline Normalised severity AUC & 5.75 & 3.50 & $3 \cdot 60^{*}$ & 1.52 & $3 \cdot 16^{*}$ & 1.98 \\
\hline
\end{tabular}

RV, rotavirus; FM, fermented milk; GFA, short-chain galacto-oligosaccharides/long-chain fructo-oligosaccharides/pectin-derived acidic oligosaccharides mixture.

${ }^{*}$ Median value was significantly different from that for RV $(P<0.05)$.

† Median value was significantly different from that for FM $(P<0.05)$

cases (Table 1). The FM group had a lower maximum diarrhoea index than the RV group $(P<0.05)$. The AUC of the severity pattern calculated during the period with diarrhoea (Table 1) was about 6 in the RV group, whereas REF animals did not develop diarrhoea and had AUC values approximately 0 (data not shown). Interestingly, a significant reduction in severity AUC (34\%) was observed for the FM group compared with the RV group, demonstrating an overall reduction in the 

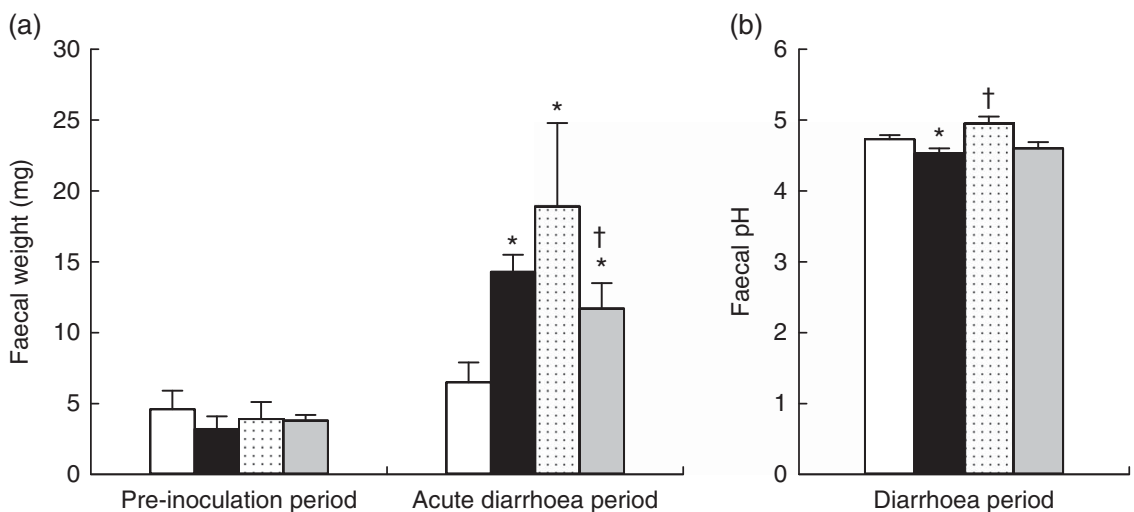

Fig. 3. Mean faecal weight ( $\mathrm{mg}$ ) from samples collected during the pre-inoculation period (before day 7) and the acute diarrhoea period (days 8-11) (a). Faecal pH from day 8-12 pooled samples (diarrhoea period) (b). Results are expressed as mean values of obtained samples from the 21 animals/group within the detailed period ( $n$ 11-62 samples per group for faecal weight and $n$ 4-6 samples per group for $\mathrm{pH}$ ), with their standard errors. Statistical significance: * $P<0.05 v$. REF; $\dagger P<0.05$ v. RV. $\square$, Reference (REF group); $\square$, rotavirus (RV group); 疄, fermented milk (FM group); $\square$, short-chain galacto-oligosaccharides/long-chain fructo-oligosaccharides/pectin-derived acidic oligosaccharides mixture (GFA group).

severity of the process $(P<0 \cdot 05)$. The GFA group showed a significant reduction in severity AUC when it was normalised (from its baseline diarrhoea index present before and after the infective process, $P<0 \cdot 05$ ).

\section{Faecal weight}

The weight of the faecal samples was recorded throughout the study. The mean faecal weight in each period was calculated in samples obtained before the inoculation day (day 7), those during the acute diarrhoea period (days 7-11) and samples belonging to the post-diarrhoea period (after day 12). Before RV inoculation, there were no differences among the groups (Fig. 3(a)); however, in the days just after RV infection, the faecal weight of animals from the RV group increased (approximately $15 \mathrm{mg}$ ) with respect to those from the REF group (approximately $6 \mathrm{mg}, P<0.05$ ). The weights of faecal samples from the FM and GFA groups were also higher than those from the REF group $(P<0 \cdot 05)$, but the GFA diet was able to decrease the faecal weight compared with the RV group $(P<0.05)$ (Fig. 3(a)). After this period, there were no differences among the groups (data not shown).

\section{Faecal pH}

Faecal samples from days 8-12 of each group were pooled and pH was measured (Fig. 3(b)). The RV group showed a lower faecal $\mathrm{pH}$ during the acute diarrhoea period with respect to the REF group $(P<0 \cdot 05)$. The FM diet avoided this acidification of faeces $(P<0.05 v$. RV). In contrast, the GFA group had an intermediate $\mathrm{pH}$ value between those of the REF group and those of the RV group.

\section{Humoral immune response}

Specific anti-RV antibodies were quantified in serum (total, IgG, IgM and IgA) (Table 2) and in intestinal washes (IgA and IgM) (Table 2) at day 14 and 21 of life. In the RV group, there were already significant levels of specific anti-RV IgA, IgG and IgM in serum and anti-RV IgA and IgM at day 14 of life. The values of each isotype in the RV group on day 14 are considered as $100 \mathrm{AU}$, and the results for the RV group on day 21 and for the other groups have been proportionally adjusted for each isotype. In the serum samples of the RV group, only anti-RV IgG titres increased significantly from day 14 to day $21(P<0 \cdot 05)$. In intestinal washes, the specific anti-RV IgA and IgM levels increased 1 week later.

Both dietary supplementations modulated the humoral immune response against the RV. In the FM group, serum anti-RV antibodies were higher than those of the RV group at day 14, which seems to be due to higher levels of specific IgM, whereas anti-RV IgA concentration was lower than that in the RV group (Table 2). In the FM group, specific IgG increased at day 21. The intestinal anti-RV IgA levels were similar to those in the RV group both at day 14 and at day 21 (Table 2). Specific total antibodies in serum from the GFA group were lower than those in the RV group at day $14(P<0 \cdot 05)$, which seems to be due to decreased levels of all anti-RV isotypes, particularly IgG, which was also reduced on day $21(P<0.05)$. In addition, in the GFA group, intestinal anti-RV IgA concentration was increased at day 14, and intestinal anti-RV IgM levels at day 21 were reduced $(P<0 \cdot 05)$.

\section{Viral shedding}

The results obtained for viral shedding after inoculation (days 7-16) can be seen in Fig. 4. The REF group (non-infected group) had a low background (in the ELISA technique). In all $\mathrm{RV}$-inoculated animals, the maximum clearance of the RV was produced on the 1 st day after inoculation (day 8). On this day, both supplemented groups had a lower value of RV shedding than that of the RV group, although it was only significant for the GFA group $(P<0.05)$, and was even similar to the background levels found in the REF group.

\section{In vitro blocking assay}

After observing the results from the viral shedding experiment, an in vitro approach was used to test the possible binding 
Table 2. Specific anti-rotavirus (RV) antibodies in serum and intestinal wash from 14- and 21-d-old rats. The values of each isotype in the RV group on day 14 are considered as 100 arbitrary units $(\mathrm{AU})$, and the results for the RV group on day 21 and for the other groups have been proportionally adjusted for each isotype

(Mean values with their standard errors; $n$ 9-12 animals per group)

\begin{tabular}{|c|c|c|c|c|c|c|c|}
\hline \multirow[b]{2}{*}{ Antibodies } & \multirow[b]{2}{*}{ Age (d) } & \multicolumn{2}{|c|}{$\mathrm{RV}(\mathrm{AU} / \mathrm{ml})$} & \multicolumn{2}{|c|}{$\mathrm{FM}(\mathrm{AU} / \mathrm{ml})$} & \multicolumn{2}{|c|}{ GFA (AU/ml) } \\
\hline & & Mean & SEM & Mean & SEM & Mean & SEM \\
\hline \multicolumn{8}{|l|}{ Serum } \\
\hline \multirow[t]{2}{*}{ Anti-RV Ig } & 14 & $100 \cdot 00$ & $17 \cdot 75$ & $154 \cdot 69^{*}$ & $21 \cdot 26$ & $66.93^{\star}$ & $18 \cdot 48$ \\
\hline & 21 & $236 \cdot 67$ & $17 \cdot 34$ & 244.89 & 13.06 & 203.44 & 4.50 \\
\hline \multirow[t]{2}{*}{ Anti-RV IgG } & 14 & $100 \cdot 00$ & 13.51 & $88 \cdot 16$ & $47 \cdot 74$ & $38.77^{*}$ & 9.92 \\
\hline & 21 & 879.79 & $227 \cdot 12$ & $1620 \cdot 86^{\star}$ & $117 \cdot 94$ & $86 \cdot 20^{\star}$ & $16 \cdot 72$ \\
\hline \multirow[t]{2}{*}{ Anti-RV IgM } & 14 & $100 \cdot 00$ & 24.96 & $162 \cdot 35$ & 41.96 & 65.85 & 10.52 \\
\hline & 21 & $153 \cdot 84$ & $48 \cdot 28$ & 95.53 & $24 \cdot 71$ & 82.66 & $10 \cdot 37$ \\
\hline \multirow[t]{2}{*}{ Anti-RV IgA } & 14 & $100 \cdot 00$ & 5.03 & $74.62^{*}$ & $7 \cdot 71$ & $83 \cdot 10$ & $8 \cdot 87$ \\
\hline & 21 & $106 \cdot 61$ & $7 \cdot 60$ & $127 \cdot 43$ & $9 \cdot 77$ & 118.41 & 9.63 \\
\hline \multicolumn{8}{|l|}{ Intestinal wash } \\
\hline \multirow[t]{2}{*}{ Anti-RV IgA } & 14 & $100 \cdot 00$ & 14.94 & 91.07 & $18 \cdot 61$ & $182.54^{*}$ & $47 \cdot 41$ \\
\hline & 21 & $239 \cdot 22$ & $32 \cdot 33$ & $195 \cdot 90$ & $22 \cdot 30$ & 166.92 & 41.06 \\
\hline \multirow[t]{2}{*}{ Anti-RV IgM } & 14 & $100 \cdot 00$ & 6.46 & $90 \cdot 31$ & $13 \cdot 76$ & $85 \cdot 29$ & $18 \cdot 69$ \\
\hline & 21 & 143.64 & $10 \cdot 89$ & 132.56 & $19 \cdot 81$ & $101 \cdot 33^{*}$ & $5 \cdot 29$ \\
\hline
\end{tabular}

FM, fermented milk; GFA, short-chain galacto-oligosaccharides/long-chain fructo-oligosaccharides/pectin-derived acidic oligosaccharides mixture. Statistical significance ${ }^{*} P<0.05 v$. RV at same time point.

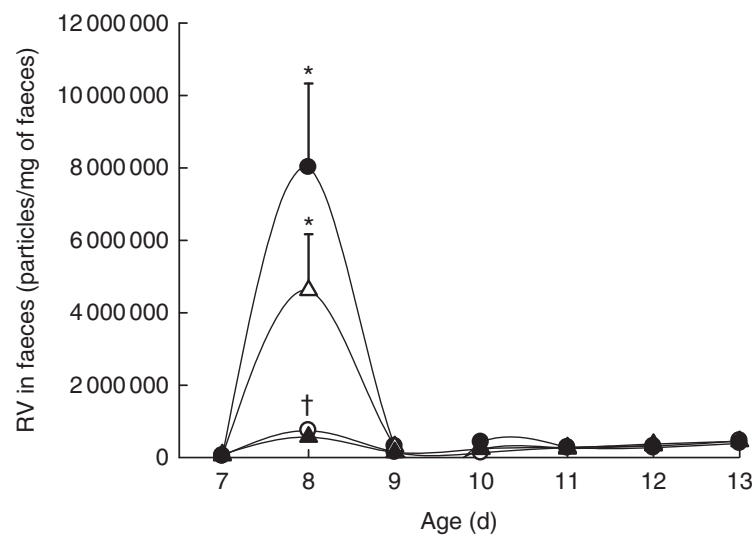

Fig. 4. Viral shedding curves of faecal samples. Results are expressed as mean values of obtained samples from the 21 animals/group within the detailed period ( $n$ 11-62), with their standard errors. Statistical significance: * $P<0.05 \mathrm{v}$. REF; † $P<0.05$ v. RV. -0 , Reference (REF group); - (RV group); $\backsim$, fermented milk (FM group); $\longrightarrow$, short-chain galactooligosaccharides/long-chain fructo-oligosaccharides/pectin-derived acidic oligosaccharides mixture (GFA group).

ability of the supplements to RV particles (Fig. 5). The number of SA-11 particles detected after incubation with several dilutions of FM and GFA products was analysed. SA-11 detection was inhibited by both products, with the inhibitory activity for both products being approximately $30 \%$ at the highest concentration assayed.

\section{Discussion}

Exclusive breast-feeding during the first 6 months of life has a protective, bifidogenic and immunomodulatory effect able to prevent or decrease the incidence and severity of RV diarrhoea among other infections ${ }^{(2)}$. In order to mimic these benefits, new supplements to be added to infant formula when infants cannot

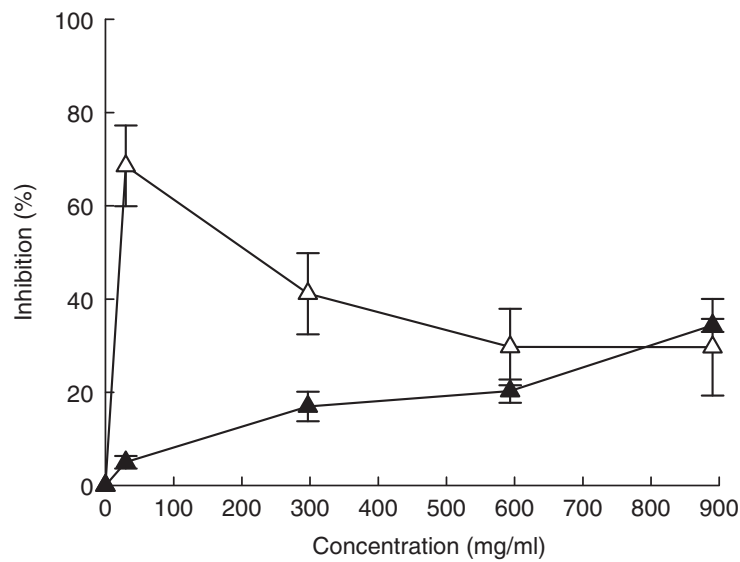

Fig. 5. Percentage of inhibition of rotavirus particle detection after incubation with fermented milk (FM) and short-chain galacto-oligosaccharides/long-chain fructo-oligosaccharides/pectin-derived acidic oligosaccharides mixture (GFA) at different dilutions of the product concentration used in the in vivo study. Values are means with their standard errors of duplicates from three independent experiments. $\triangle$, FM; $\neg$, GFA.

be breast-fed have been recently investigated. In the present study, both products based on microbial/prebiotic concepts (FM and GFA) have been evaluated on a model of RV-induced diarrhoea in suckling rats.

FM administration was able to reduce clinical diarrhoea in terms of incidence, duration and severity. A reduction in diarrhoea severity had already been reported in a germ-free suckling rat RV model supplemented with milk fermented by a Lactobacillus casei strain ${ }^{(25)}$, and also in infants supplemented with a cell-free fermented infant formula ${ }^{(26)}$. The latter study demonstrated that the presence of live micro-organisms is not essential to obtain probiotic-like effects. The FM also avoided the RV-induced acidification of faeces. However, this result, similar to that found in the REF group, contrasts with the reduction of faecal $\mathrm{pH}$ in healthy mice and humans receiving 
a cell-free fermented formula ${ }^{(23,24)}$. This could be due to the effect of breast-feeding in our study, which may already acidify the intestinal content. In RV-induced groups, viral shedding was mainly produced on day 8 , agreeing with previous studies using this model ${ }^{(27)}$. However, in the FM group, viral shedding was slightly lower than that in the RV group, which suggests that the FM has a certain capacity to bind the RV particles. In fact, interactions between enterocyte surface glycan and VP7/VP4 RV proteins are crucial for infection ${ }^{(32)}$, and glycoproteins have been characterised as active compounds of a $\mathrm{FM}^{(23)}$. In consequence, it seems reasonable to think that some FM components could bind the virus. This is why we performed the blocking assay, which showed an inhibition of approximately $30 \%$ of virus detection in the presence of this FM.

The GFA group showed a softer consistency of faecal samples throughout the study, which were even sometimes considered as diarrhoeic. This effect has already been described for some prebiotics, and it is actually useful to make formula-fed infants' faeces more similar to those from breast-fed infants and to reduce constipation ${ }^{(5,6,33)}$. However, the difficulty of differentiating the softer stool consistency induced by the probiotic from the RV-induced diarrhoea is a limitation of this suckling rat model. In spite of that, this diet showed clinical improvements similar to those of FM administration and in line with other studies, where a specific mixture of scGOS/lcFOS supplementation prevented infections in infants ${ }^{(5,13,14,34)}$. Although the faeces were scored in a blinded manner, faecal weight seemed to be a more objective measure of the water content in the faeces. In this context, a reduction in faecal weight in the acute diarrhoea period in the group supplemented with GFA also evidenced the improvement induced by this diet. In addition, the faecal $\mathrm{pH}$ in the GFA group had an intermediate value between those of the REF and RV groups. This fact does not discard that this product may avoid the RV-induced electrolytic disorder, but a reduction of $\mathrm{pH}$ could be because of the acidity of pAOS and/or caused by scGOS/lcFOS directly. The increase in bacterial fermentation can lead to an increase in SCFAs production, and thus $\mathrm{pH}$ reduction. In fact, lower $\mathrm{pH}$ has been reported as a beneficial effect associated with prebiotic oligosaccharides $^{(7,33)}$. In the group supplemented with GFA, viral shedding was greatly reduced; in fact, it was comparable with that of the REF group. The blocking assay indicated a certain inhibition in detecting the virus in the presence of GFA, similarly to FM, which may indicate a lower infection of the epithelial cells in the intestine and a lower replication. In this sense, acidic oligosaccharides are known to be able to interact with the epithelial surface and to prevent the pathogen adhesion $^{(3)}$.

Overall, both concepts tested showed protective effects against RV-induced diarrhoea in suckling rats. A possible mechanism responsible for the reduction of clinical signs produced by both concepts could be the direct interaction with viral particles and their blocking, avoiding the entry into enterocytes and, consequently, their replication. However, we cannot discard the possibility that the concepts could also interact with the intestinal microbiota, or directly with the cell surface, stimulating changes in the mucus and reinforcing the epithelial barrier.
Moving to the immune response against $\mathrm{RV}$, this infection induces local and systemic, humoral and cellular immune responses. B cells presenting the $\alpha 4 \beta 7$ homing integrin and $\mathrm{T} \mathrm{CD} 4^{+}$cells are crucial for the specific antibodies production, and $\mathrm{T} \mathrm{CD} 8^{+}$cells constitute the cell-mediated immune response, which by means of inducing cytotoxicity shortens the infection and promotes viral clearance. Similar to most humoral immune responses, specific anti-RV antibodies released are first IgM and then IgG and IgA isotypes, with the levels of anti-RV IgA, either intestinal or systemic, being better correlated with protection $^{(35-37)}$. Nevertheless, the acquirement of protection against RV is not fully understood ${ }^{(35-37)}$. In the present study, the RV infection caused an increase of specific antibodies in serum and intestinal wash samples. The FM diet did cause some changes in anti-RV antibodies concentration. Some studies have suggested that the increase in intestinal IgA could be the mechanism of action of $\mathrm{FM}^{(26)}$, but our results do not agree with that suggestion because intestinal IgA levels in the FM group were similar to those found in the RV group. On the other hand, the GFA diet's results suggest an enhancement in the early response as shown in intestinal IgA, which agrees with other studies showing that GFA activated the immune response ${ }^{(17-21)}$, and allows us to hypothesise that these antibodies may solve the infection rapidly, with fewer antibodies needed later on. This early increase in intestinal anti-RV IgA synthesis seems to have been enough to fight against the virus.

In conclusion, both microbial/prebiotic-related concepts tested here showed some beneficial effects on RV-induced diarrhoea in a neonatal rat model and can be considered for inclusion in infant formulae or supplements. Further studies are needed to understand their mechanism of action. A more in-depth investigation into the direct interaction of the products with the virus would be of interest, as well as their effect on the intestinal microbiota.

\section{Acknowledgements}

This study was supported by a Nutricia Research grant in collaboration with the Fundació Bosch i Gimpera (FBG306349).

The authors' contributions were as follows: F. J. P.-C., A. F., M. C., K. V. L., K. K., J. G. and J. K. designed the study; M. R. A., T. P.-B. and S. R.-R. conducted the study and acquired data; F. J. P.-C. and M. R. A. reviewed the data, interpreted the results and drafted the manuscript; all the authors contributed to the critical review and revision of the manuscript.

The authors declare that they have a financial relationship with the organisation that sponsored the research. K. V. L., K. K., J. G. and J. K. are employees of Nutricia Research B.V. The other authors declare that they have no conflicts of interest.

\section{References}

1. World Health Organization \& UNICEF (2003) Global Strategy for Infant and Young Child Feeding. Geneva: WHO. http:// apps.who.int/iris/bitstream/10665/42590/1/9241562218.pdf? ua $=1 \& u a=1$ (accessed May 2016). 
2. Krawczyk A, Lewis MG, Venkatesh BT, et al. (2016) Effect of exclusive breastfeeding on rotavirus infection among children. Indian J Pediatr 83, 220-225.

3. Jeurink PV, Van Esch BCAM, Rijnierse A, et al. (2013) Mechanisms underlying immune effects of dietary oligosaccharides. Am J Clin Nutr 98, 572-577.

4. Pérez-Cano FJ, Marín-Gallén S, Castell M, et al. (2008) Supplementing suckling rats with whey protein concentrate modulates the immune response and ameliorates rat rotavirusinduced diarrhea. J Nutr 138, 2392-2398.

5. Salvatore S \& Vandenplas Y (2010) Prebiotics and probiotics in therapy and prevention of gastrointestinal diseases in children. In Bioactive Foods in Promoting Health, pp. 181-203 [RR Watson and VR Preedy, editors]. Amsterdam: Elsevier.

6. Veereman-Wauters G, Staelens S, Van de Broek H, et al. (2011) Physiological and bifidogenic effects of prebiotic supplements in infant formulae. J Pediatr Gastroenterol Nutr 52, 763-771.

7. Djouzi Z \& Andrieux C (1997) Compared effects of three oligosaccharides on metabolism of intestinal microflora in rats inoculated with a human faecal flora. Br J Nutr 78, 313-324.

8. Pan X, Chen F, Wu T, et al. (2009) Prebiotic oligosaccharides change the concentrations of short-chain fatty acids and the microbial population of mouse bowel. J Zhejiang Univ Sci B 10, 258-263.

9. Bakker-Zierikzee AM, Van Tol EAF, Kroes H, et al. (2006) Faecal SIgA secretion in infants fed on pre- or probiotic infant formula. Pediatr Allergy Immunol 17, 134-140.

10. Scholtens PAMJ, Alliet P, Raes M, et al. (2008) Fecal secretory immunoglobulin A is increased in healthy infants who receive a formula with short-chain galacto-oligosaccharides and long-chain fructo-oligosaccharides. J Nutr 138, 1141-1147.

11. Bryk G, Coronel MZ, Pellegrini G, et al. (2015) Effect of a combination GOS/FOS ${ }^{\circledR}$ prebiotic mixture and interaction with calcium intake on mineral absorption and bone parameters in growing rats. Eur J Nutr 54, 913-923.

12. Bryk G, Hernandez E, Chaves MG, et al. (2015) Utilidad de una mezcla prebiótica para aumentar la absorción y retención de calcio durante el crecimiento normal y durante la recuperación de una malnutrición proteica: modelo experimental en ratas (Usefulness of a prebiotic mixture to increase calcium absorption and retention during normal growth and during recovery from protein malnutrition: experimental model in rats). Actual Osteol 11, 19-37.

13. Arslanoglu S, Moro GE \& Boehm G (2007) Early supplementation of prebiotic oligosaccharides protects formula-fed infants against infections during the first 6 months of life. J Nutr 137, 2420-2424.

14. Bruzzese E, Volpicelli M, Squeglia V, et al. (2009) A formula containing galacto- and fructo-oligosaccharides prevents intestinal and extra-intestinal infections: an observational study. Clin Nutr 28, 156-161.

15. Osborn DA \& Sinn JKH (2013) Prebiotics in infants for prevention of allergy. The Cochrane Database of Systematic Reviews, issue 3, CD006474.

16. Garthoff JA, Heemskerk S, Hempenius RA, et al. (2010) Safety evaluation of pectin-derived acidic oligosaccharides (pAOS): genotoxicity and sub-chronic studies. Regul Toxicol Pharmacol 57, 31-42.

17. Gori A, Rizzardini G, Van't Land B, et al. (2011) Specific prebiotics modulate gut microbiota and immune activation in HAART-naive HIV-infected adults: results of the "COPA" pilot randomized trial. Mucosal Immunol 4, 554-563.

18. Schijf MA, Kruijsen D, Bastiaans J, et al. (2012) Specific dietary oligosaccharides increase Th1 responses in a mouse respiratory syncytial virus infection model. $J$ Virol 86, 11472-11482.

19. Vos AP, Haarman M, VanGinkel JWH, et al. (2007) Dietary supplementation of neutral and acidic oligosaccharides enhances Th1-dependent vaccination responses in mice. Pediatr Allergy Immunol 18, 304-312.

20. Kerperien J, Jeurink PV, Wehkamp T, et al. (2014) Non-digestible oligosaccharides modulate intestinal immune activation and suppress cow's milk allergic symptoms. Pediatr Allergy Immunol 25, 747-754.

21. Grüber C, van Stuijvenberg M, Mosca F, et al. (2010) Reduced occurrence of early atopic dermatitis because of immunoactive prebiotics among low-atopy-risk infants. J Allergy Clin Immunol 126, 791-797.

22. Granier A, Goulet O \& Hoarau C (2013) Fermentation products: immunological effects on human and animal models. Pediatr Res 74, 238-244.

23. Romond MB, Ais A, Yazourh A, et al. (1997) Cell-free wheys from bifidobacteria fermented milks exert a regulatory effect on the intestinal microflora of mice and humans. Anaerobe 3, 137-143.

24. Romond MB, Ais A, Guillemot F, et al. (1998) Cell-free whey from milk fermented with bifidobacterium breve C50 used to modify the colonic microflora of healthy subjects. J Dairy Sci 81, 1229-1235.

25. Guérin-Danan C, Meslin JC, Chambard A, et al. (2001) Food supplementation with milk fermented by Lactobacillus casei DN-114 001 protects suckling rats from rotavirus-associated diarrhea. J Nutr 131, 111-117.

26. Thibault H, Aubert-Jacquin C \& Goulet O (2004) Effects of long-term consumption of a fermented infant formula (with Bifidobacterium breve c50 and Streptococcus thermophilus 065) on acute diarrhea in healthy infants. J Pediatr Gastroenterol Nutr 39, 147-152.

27. Pérez-Cano FJ, Castell M, Castellote C, et al. (2007) Characterization of clinical and immune response in a rotavirus diarrhea model in suckling Lewis rats. Pediatr Res 62, 658-663.

28. Pérez-Cano FJ, Franch A, Castellote C, et al. (2012) The suckling rat as a model for immunonutrition studies in early life. Clin Dev Immunol 2012, 537310.

29. Parashar UD, Hummelman EG, Bresee JS, et al. (2003) Global illness and deaths caused by rotavirus disease in children. Emerg Infect Dis 9, 565-572.

30. Reeves PG, Nielsen FH \& Fahey GC (1993) AIN-93 purified diets for laboratory rodents: final report of the American Institute of Nutrition ad hoc writing committee on the reformulation of the AIN-76A rodent diet. J Nutr 123, 1939-1951.

31. Pérez-Cano FJ, Marín-Gallén S, Castell M, et al. (2007) Bovine whey protein concentrate supplementation modulates maturation of immune system in suckling rats. Br J Nutr $\mathbf{9 8}$, S80-S84.

32. Etzold S \& Bode L (2014) Glycan-dependent viral infection in infants and the role of human milk oligosaccharides. Curr Opin Virol 7, 101-107.

33. Westerbeek E, Hensgens R, Mihatsch W, et al. (2011) The effect of neutral and acidic oligosaccharides on stool viscosity, stool frequency and stool $\mathrm{pH}$ in preterm infants. Acta Paediatr 100, 1426-1431.

34. Rigo-Adrover M, Saldaña-Ruíz S, van Limpt K, et al. (2016)

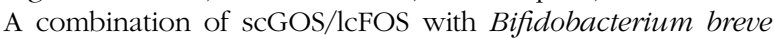
$\mathrm{M}-16 \mathrm{~V}$ protects suckling rats from rotavirus gastroenteritis. Eur J Nutr (epublication ahead of print version 25 April 2016).

35. Desselberger U \& Huppertz HI (2011) Immune responses to rotavirus infection and vaccination and associated correlates of protection. J Infect Dis 203, 188-195.

36. Greenberg HB \& Estes MK (2009) Rotaviruses: from pathogenesis to vaccination. Gastroenterology 136, 1939-1951.

37. Riechmann ER, Cilleruelo ML \& Rivero MJ (2006) Infección por rotavirus en España (Rotavirus infection in Spain). In Infección por rotavirus (Rotavirus Infection), pp. 67-75 [ER Riechmann, editor]. Madrid: Undergraf S.L. 